\title{
HIGH-REDSHIFT OBJECTS AS PROBES OF NEARBY COSMIC VOIDS
}

(Letter to the Editor)

NOAH BROSCH and J. MAYO GREENBERG

Laboratory Astrophysics, Huygens Laboratorium, University of Leiden, The Netherlands

(Received 18 October, 1982)

\begin{abstract}
We show that present day observational techniques make it possible to detect diffuse gas masses down to $\sim 10^{10} M_{\odot}$ in cosmic voids with linear sizes of $\sim 1 \mathrm{Mpc}$ or larger. The observations consist of searches for absorption lines, similar in character to those produced in galactic interstellar clouds, in the spectra of high-redshift objects located beyond previously identified voids adjoining nearby superclusters.
\end{abstract}

\section{Introduction}

Recent observations of the three-dimensional distribution of galaxies show that the vast majority are concentrated as strings comprising groups and clusters of galaxies. Neighboring strings bunch up into entities called superclusters which, however, fill up only a small fraction, about $10 \%$ or less, of the available volume. The remaining spaces, hereafter called 'voids', contain almost no luminous matter and have typical sizes of the order of a few Mpc. They may constitute more than $90 \%$ of the total volume of the Universe (Zeldovich et al., 1982).

The question - whether the lack of luminous matter implies a dearth of ass in general - is crucial to most cosmological theories. This is because theories of cosmology depend on gravitational mass rather than luminous matter. Thus it is important to determine if and to what density the voids are filled with optically invisible objects. Some evidence for such material already exists for single galaxies (flat rotation curves) and for groups and clusters (virial mass). Perhaps it is present on larger scales as well.

Looking for dark matter in voids has an advantage over searches in galactic envelopes because of the enormous path lengths involved. Furthermore, choosing voids identified beforehand sets limits on the redshift ranges to be searched, in contrast to identifications of members in the 'L $\alpha$-forest' (Sargent et al., 1980). In this paper we address ourselves to a possible observation which can put some limits on the mass available in some voids.

\section{Proposed Observations}

At least four nearby voids have been identified in the space distribution of galaxies. These are given in Table I with their dimensions and references. The QSO catalogue (Hewitt and Burbidge, 1979) lists a number of bright $(V \leq 16.0)$ objects seen beyond the voids defined in Table I. These QSO's and BL Lac objects are given in Table II. 
TABLE I

Nearby voids

\begin{tabular}{|c|c|c|c|c|c|}
\hline \multirow[t]{2}{*}{ Name } & \multicolumn{4}{|c|}{ Approximate limits } & \multirow[t]{2}{*}{ References } \\
\hline & in $\alpha$ & & in $\delta$ & in $v_{\odot}\left(\mathrm{km} \mathrm{s}^{-1}\right)$ & \\
\hline Perseus-Pisces & $0^{\text {h }}$ & $\rightarrow 2$ & $+0^{\circ} \rightarrow+20^{\circ}$ & $6500 \rightarrow 9500$ & Einasto et al. (1980) \\
\hline Coma/A1367 & $12^{\mathrm{h}}$ & $\rightarrow 13$ & $+20^{\circ} \rightarrow+30^{\circ}$ & $4800 \rightarrow 6300$ & Gregory and Thompson (1978) \\
\hline Bootes & $13^{\mathrm{h}}$ & $\rightarrow 16$ & $+40^{\circ} \rightarrow+60^{\circ}$ & $12000 \rightarrow 18000$ & Shectman (1983) \\
\hline Hercules & $15^{\mathrm{h}} 52^{\mathrm{r}}$ & ${ }^{\mathrm{m}} \rightarrow 16^{\mathrm{h}} 12^{\mathrm{m}}$ & $+14^{\circ} \rightarrow+22^{\circ}$ & $5500 \rightarrow 8500$ & Tarenghi et al. (1979) \\
\hline
\end{tabular}

TABLE II

Bright QSO's and BL Lac's towards voids

\begin{tabular}{lll}
\hline Void & QSO/BL Lac & $V$ \\
\hline Perseus-Pisces & UM 202 & 16.0 \\
& III Zw 2 & 15.4 \\
& 0026+129 & 14.8 \\
& I Zw 1 & 14.1 \\
Coma/A1367 & GQ Comae & 15.5 \\
& ON 325 & 15.5 \\
& Ton 616 & 16.0 \\
& 12225+317 & 15.9 \\
& Ton 1542 & 15.3 \\
Bootes & 1351 +640 & 14.8 \\
& 4 C 58.29 & 16.0 \\
& OQ 530 & 15.0 \\
& 1435+630 & 15.0 \\
& 4 C 37.43 & 15.5 \\
\hline
\end{tabular}

Note to Table II: Hewitt and Burbidge (1979) list many QSO's beyond the Hercules void but give no magnitudes for them. It is to be hoped that some are bright enough for a study like that proposed here.

\section{TABLE III}

Some candidate transitions for the search

\begin{tabular}{lll}
\hline Species & $\lambda_{0}(\AA)$ & $10^{6} \eta f$ \\
\hline $\mathrm{NV}$ & 1238 & 1.50 \\
$\mathrm{Si} I V$ & 1393 & 1.67 \\
$\mathrm{C}$ IV & 1549 & 6.29 \\
$\mathrm{Al}$ III & 1854 & 0.12 \\
$\mathrm{Mg}$ II & 2795 & 1.63 \\
$\mathrm{Mg}$ I & 2852 & 4.21 \\
$\mathrm{Ca} I I$ & 3933 & 0.14 \\
$\mathrm{Ca}$ I & 4226 & 0.35 \\
$\mathrm{Fe}$ I & 4383 & 1.02 \\
$\mathrm{Na}$ I & 5889 & 0.12 \\
$\mathrm{Si} I I$ & 6347 & 2.81 \\
$\mathrm{Ne}$ I & 6402 & 3.95 \\
\hline
\end{tabular}


We propose that good high-resolution $(\sim(\AA)$ optical spectrophotometry, as well as spectrophotometry in ultraviolet, be obtained to search for absorption lines formed in the intergalactic media of these voids. The resolution is required to separate absorptions produced in voids from close foreground and background features.

We will now show that such observations, even though not easily obtained, are feasible with large telescopes and state-of-the-art detectors, like the IPCS, or similar ones.

Consider a void in front of a source which produces essentially a featureless continuum. Let the path length through the void be $D$ and the number density of hydrogen atoms be $n_{\mathrm{H}}$, assumed for simplicity to be uniform. Assume also that an absorption line at laboratory wavelength $\lambda_{0}$ is produced by species $\mathrm{X}$ of relative number abundance $\eta=n_{\mathrm{X}} / n_{\mathrm{H}}$. It is reasonable to assume, despite the large path length expected, that the absorption across the line will be optically thin because of the probably low $n_{\mathrm{H}}$ and the presumably large Doppler width. Thus, as shown in Spitzer (1978), the equivalent width of the absorption line will be given by

$$
W(\AA)=0.7\left\{\frac{n_{\mathrm{H}}}{10^{-6} \mathrm{~cm}^{-3}}\right\}\left\{\frac{D}{\mathrm{Mpc}}\right\}\left\{\frac{\lambda_{0}}{5000 \AA}\right\}^{2}\left\{\frac{\eta f}{10^{-6}}\right\},
$$

where $f$ is the oscillator strength of the absorption transition. Note that a volume $1 \mathrm{Mpc}$ on a side containing $10^{-6} \mathrm{H}$ atom per cubic centimeter has a mass of $\sim 10^{10} M_{\odot}$ and a column density of $\sim 3 \times 10^{18} \mathrm{~cm}^{-2}$. It is clear that good candidate lines for such a search are those absorptions from the ground state that combine a large relative abundance with strong oscillator strength. A number of possible candidates are listed in Table III together with their $\eta f$ products. There we have let the elemental abundance in the voids be 0.1 of the cosmic abundances as given in Allen (1973) and assumed that the given atom is in only one ionization state. We have excluded $\mathrm{L} \alpha$ because, for the nearby voids, it cannot be resolved from the geocoronal emission in IUE spectra.

It is possible that absorption lines produced in some foreground voids have already been observed (even though not recognized as such) in spectra of high redshift objects. The QSO $0026+129$ shows features corresponding to the signature of some proposed candidates in Table III, redshifted by the average velocity of the foreground Perseus-Pisces void (spectrum appearing in Puetter et al., 1978). The same is true for $1225+137$, seen behind the Coma/A1365 void (spectrum published by Puetter et al., 1979). However, the confirmation that these features are absorption lines produced in the specific voids would come not only from searching within a predetermined velocity range but also from the appearance of similar absorptions at nearly the same wavelength in spectra of other nearby quasars or BL Lac objects like those proposed in Table II. Such associated features are possibly present in the spectra of $1222+228,1225+317$, and $1247+267$ published by Young et al. (1982).

Finally, the classical Gunn-Peterson test (Peebles, 1971) does not conflict with the observations proposed here because it measures a limit on the amount of neutral hydrogen at cosmological distances $z>2.0$ ). The physical conditions in the evolving 
universe might then have been completely different from those encountered today, in nearby voids.

\section{Conclusions}

We have shown that the possible important cosmological parameter measuring the existence of matter present in the cosmic voids can be determined from straightforward observations of absorption of absorption lines in the spectra of QSO's and BL Lac objects beyond pre-identified voids. Spectrophotometric observations with resolution

$\sim 1 \AA$ and good $\mathrm{S} / \mathrm{N}$ should show absorption features with typical equivalent width of the order of angstroms.

\section{Acknowledgements}

We acknowledge discussions on this subject with W. Bijleveld, Y. Hoffman, H. C. van de Hulst, N. Krumm, C. Norman, J. H. Oort, M. Schmidt, and W. W. Shane. We thank the many Leiden colleagues who have read and commented on the manuscript.

\section{References}

Allen, C. W.: 1973, Astrophysical Quantities, 3rd edition, The Athlone Press, University of London. Einasto, J., Jôeveer, M., and Saar, E.: 1980, Monthly Notices Roy. Astron. Soc. 193, 353.

Gregory, S. A. and Thompson, L. A.: 1978, Astrophys. J. 222, 784.

Hewitt, A. and Burbidge, G.: 1979, Astrophys. J. Suppl. 43, 57.

Peebles, P. J. E.: 1971, Physical Cosmology, Princeton University Press, Princeton, New Jersey.

Puetter, R. C., Smith, H. E., Soifer, B. T., Willner, S. P., and Pipher, J. L.: 1978, Astrophys. J. 226, L53.

Puetter, R. C., Smith, H. E., and Willner, S. P.: 1979, Astrophys. J. 227, L5.

Sargent, W. L. W., Young, P. J., Boksenberg, A., and Tytler, R. F.: 1980, Astrophys. J. Suppl. $42,41$.

Savage, B. D. and de Boer, K. S.: 1981, Astrophys. J. 243, 460.

Shectman, S. A.: 1982, paper presented at the IAU Symp. 104.

Spitzer, L.: 1978, Physical Processes in the Interstellar Medium, Wiley and Sons, New York, p. 52.

Tarenghi, M., Tifft, W. G., Chincarini, G., Rood, H. J., and Thompson, L. A.: 1979, Astrophys. J. $234,793$.

Young, P., Sargent, W. L. W., and Boksenberg, A.: 1982, Astrophys. J. Suppl. 48, 455.

Zeldovich, Ya. B., Einasto, J., and Shandarin, S. F.: 1982, Tallin Preprint A-2. 